\title{
A Complete Time-Dependent Carrier-borne Epidemic Model
}

\author{
Gurprit Grover', Vajala Ravi', Vishal Deo ${ }^{3}$, Chandra Bhan Yadav ${ }^{4}$ \\ 1,4 Department of Statistics, Delhi University, New Delhi, India. \\ ${ }^{2}$ Department of Statistics, Lady Shri Ram College, Delhi University, New Delhi, India. \\ ${ }^{3}$ Department of Statistics, Ramjas College, Delhi University, New Delhi, India. \\ DOI: https://doi.org/10.24321/0019.5138.202171
}

\section{I $\quad \mathbf{N} \quad \mathbf{F} \quad \mathbf{O}$}

\section{Corresponding Author:}

Chandra Bhan Yadav, Department of Statistics,

Delhi University, New Delhi, India.

E-mail Id:

chandrabhansitare@gmail.com

Orcid Id:

https://orcid.org/0000-0002-5021-7051

How to cite this article:

Grover G, Ravi V, Deo V, Yadav CB. A Complete

Time-Dependent Carrier-borne Epidemic Model.

J Commun Dis. 2021;53(4):29-35.

Date of Submission: 2021-09-17

Date of Acceptance: 2021-12-07

\section{$\begin{array}{llllllll}\mathbf{A} & \mathbf{B} & \mathbf{S} & \mathbf{T} & \mathbf{R} & \mathbf{A} & \mathbf{C} & \mathbf{T}\end{array}$}

A complete time-dependent carrier-borne epidemic model in the presence of more than one carrier is developed in this article. Also, the probability of survival of susceptibles for a random infectious time period is derived. Moreover, the maximum likelihood estimation (MLE) of relative infection rate is obtained which is found to be more efficient than the one obtained by Roy Sunders \& Richard J Kryscio in 1976. The proposed complete time-dependent carrierborne epidemic model gives a better explanation than the stochastic model suggested by Wiess in 1965.

Keywords: Carrier Borne Epidemic Model, MLE, Sampling Distribution, Consistency, Efficiency, Infection rate, Removal Rate

\section{Introduction}

Studies on epidemic models have been going on for over 100 years and multitudes of mathematical (deterministic) and statistical (stochastic) epidemic models have been developed which mainly serve the purpose of predictions related to the spread of different kinds of epidemics. In this article, we are focusing on one of the most important classes of epidemic models, called the carrier-borne epidemic model. Carrier-borne diseases are contagious diseases caused by pathogens, like viruses, protozoa and bacteria, which are communicated by humans, or animal agents known as carriers. Basically, carriers are infectives without visible symptoms of the diseases and play a very significant role in the spread of an epidemic, for example, pathogens such as hepatitis B virus, herpes simplex virus, and HIV are frequently transmitted by asymptomatic human carriers.
In 1991, Ross published a report on the prevention and the transmission of malaria. ${ }^{1} \mathrm{AJ}$ Lotka set up a system of differential equations to represent the presumptive course of events in the development of an endemic in which recovery is accompanied by acquired immunity for malaria in 1923. ${ }^{2}$ GS Wilson discussed measles immunisation at an international conference in $1962 .{ }^{3} \mathrm{~T}$ The first work on the stochastic epidemic model is believed to be published by McKendrick in $1926 .{ }^{4}$ However, it went unnoticed in the academic community for a very long time, till MS Bartlett extended the work of McKendrick in the year $1946 .{ }^{5}$ The foundations of carrierborne epidemic models were laid down by GH Weiss through his pioneer work published in the year $1965 .{ }^{6} \mathrm{He}$ developed the overall deterministic formulation of the carrier-borne epidemic model and presented a stochastic framework for estimating the ultimate size of a carrierborne epidemic using the concept of Markov process. 
To define his model, Weiss assumed that all carriers can be identified and isolated using the available public health measures. Also, Weiss considered an epidemic in a closed population where infections are spread by initially introduced carriers and no new carriers in the form of infectives are introduced in the population.

This simple model of Weiss has been extended further by many authors, under different assumptions regarding the carriers and the type of population. Downton 7,8 introduced a new stochastic epidemic model which assumed that apart from the initially introduced carriers, new carriers can be created from the susceptibles in the population. In general, this is a more realistic scenario for a carrier-borne epidemic, and, in that sense, Downton's model can be considered as a generalisation over Weiss's model. Severo ${ }^{9}$ further extended the model given by Downton by allowing the removal of both susceptibles and infectives from the population. He also provided a recursive procedure to estimate state probabilities at any given time, which could be further utilised to obtain maximum likelihood estimates of the model parameters. Further work in this model was done by Daniels, ${ }^{10}$ who provided an exact relation between the probability that a specific number of susceptibles remain uninfected at the end of an epidemic, and the probability that in another epidemic starting with the total number of susceptibles equal to the total number of infected in the previous epidemic, all susceptibles will get infected. Further extension of the model given by Weiss ${ }^{6}$ was provided by Becker. ${ }^{11}$ He generalised the carrier-borne epidemic model to allow for variation in the susceptibility of the individuals and for variation in the infective capacity of the carriers. Jerwood ${ }^{12}$ proposed evaluation of the cost of a carrier-borne epidemic as a sum of two components - total area generated by the trajectory of the carriers, and total cost of isolating susceptibles and identified carriers. He used the model devised by Downton $(1967)^{8}$ to derive the components of the cost function. As another important extension of Weiss's model, Routleff ${ }^{13}$ developed a carrier-borne epidemic model. In this model, along with the number of carriers present, carrier removal is also dependent on the number of affected susceptibles. This was because a search team for carriers will not be active till new infections are reported from a region. Lefevre $C$ and Malice MP (1988) ${ }^{14}$ compared the progression of the disease in the case of heterogeneous and homogeneous populations in order to investigate the impact of differences in infection and removal rates on Weisstype carrier-borne epidemic models. Clancy D (1966) $)^{15}$ gave a carrier-borne epidemic model incorporating population mobility. Dietz K (1966) ${ }^{16}$ explained how only the carriers are responsible for spread of the disease. Ball $F(1985)^{17}$ defined deterministic and stochastic models of epidemics with several kinds of susceptibles. Also, Ball $F$ and Clancy $D$ revealed how to get the final outcome and temporal solution of a carrier-borne epidemic model in $1985 .{ }^{18} \mathrm{Gani}$ gave the general stochastic model of epidemic in $1967 .{ }^{19} \mathrm{He}$ also defined an extension of the classical carrier-borne epidemic model for two stages of HIV infection in $1991 .{ }^{20}$ Picard and Lefevre ${ }^{21}$ further generalised this model in 1991 for multiple stages of infection. Gani used a classical Markov chain approach, whereas Picard and Lefevre used a martingale approach, to obtain the joint probability function of the numbers of susceptibles, infectives, and carriers present at any time in the population. W Henderson $(1979)^{22}$ presented an alternative approach to derive a general class of results in carrier-borne epidemic theory. R Watson (1980) ${ }^{23}$ used random time scale transformations to obtain equations and to derive asymptotic approximation for the size distribution.

In this article, we have proposed a complete timedependent probabilistic formulation of the existing carrier-borne epidemic model given by Weiss, ${ }^{6}$ as opposed to its widely used stochastic process formulation. The infectious time period has been treated as a random variable having a probability distribution. Using the results of simple death process of Bharucha Reid (1960), ${ }^{24}$ the expression for conditional probability of survival of $k$ out of $m$ susceptibles, in the presence of $n$ carriers, after a given infectious time period has been derived. The expression for the probability that a population of $m$ susceptibles are reduced to $\mathrm{k}$ survivors at the termination of an epidemic initiated by $n$ carriers is also derived and the result matches that of Weiss. ${ }^{6}$ Further, we have obtained the method of moment estimator (MME) and maximum likelihood estimator (MLE) of the relative infection rate. Saunders and Kryscio ${ }^{25}$ also provided MLE of the relative infection rate corresponding to Weiss's carrier-borne epidemic model. Weiss ${ }^{27}$ and Saunders and Kryscio ${ }^{25}$ applied their results to the data from a typhoid epidemic in Zermatt, Switzerland. To compare the performance of our estimates with that of Saunders and Kryscio, ${ }^{25}$ we have computed the MMEs and MLEs of the relative infection rate for the same data, at three different values of $n$. Standard errors of the MLEs have also been computed to assess the efficiency of these estimates as compared to those of Saunders and Kryscio. ${ }^{25}$ Values of conditional probabilities computed at different time points, for different combinations of a number of susceptibles and number of carriers, are presented in tables and figures to provide further insight into the behaviour of the timedependent carrier-borne epidemic model. 


\section{Development of Complete Time-Dependent Carrier-borne Epidemic Model}

Suppose that an epidemic is initiated by $n$ carriers (where $n \geq 1$ ) in the presence of $m$ susceptibles. It is assumed that the epidemic can terminate in one of the two ways - either all carriers are eliminated or the entire population gets infected from the disease. Let $\pi_{k} \mid \tau(m, n)$ to be the probability of survival of $k$ susceptibles out of $m$ susceptibles during infectious time period $\tau$. The $\pi_{k \mid \tau}(m, n)$ satisfies the equation.

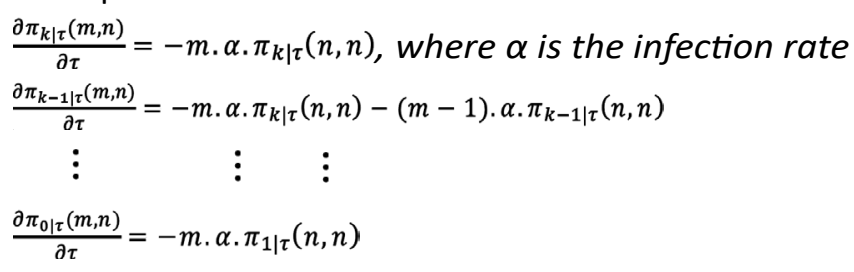

Which is nothing but a simple death process with the following solution.

$\pi_{k \mid \tau}(m, n)=\left(\begin{array}{l}m \\ k\end{array}\right) \cdot\left(e^{-\alpha \tau}\right)^{k} \cdot\left(1-e^{-\alpha \tau}\right)^{m-k}$

Further, we define relative infection rate $\sigma$ as $\sigma=\frac{\alpha}{\beta}$, where $B$ is the recovery/ removal rate

Now, to estimate the unknown parameters, we define the following random variables.

$K$ : A random variable representing the number of susceptibles (a discrete random variable).

$T$ : A random variable representing the infectious time period (a continuous random variable).

The infectious time period $T$ is assumed to follow gamma distribution with parameters ' $n$ ' (shape) and $\beta$ (scale). For computational convenience, let us consider the transformed infectious time period as $T=\mathcal{T}$. $\beta$, so that the transformed infectious time period $T$ follows gamma(n,1) with the following probability density function.

$f(t)=\frac{1}{\Gamma(n)} \cdot t^{n-1} \cdot e^{-t} ;$ Where $t>0$

Let $\pi_{k, t}(m, n)$ be the probability that $\mathrm{k}$ out of $\mathrm{m}$ susceptibles survive after the infectious time period $t$, when the epidemic starts with $\mathrm{n}$ carriers. Using equations (1) and (3), $\pi_{k, t}(m, n)$ is obtained as the joint distribution of $\mathrm{K}$ and $\mathrm{T}$ as follows.

$\pi_{k, t}(m, n)=P[K=k \mid T=t] \cdot f(t)=\left(\begin{array}{l}m \\ k\end{array}\right)\left(e^{-\sigma t}\right)^{k}\left(1-e^{-\sigma t}\right)^{m-k} \frac{1}{\Gamma(n)} t^{n-1} e^{-t}$

Where $\mathrm{t}>0$ and $\mathrm{k}=0,1,2, \ldots, \mathrm{m}$;

Putting $\mathrm{n}=1$ in equation (4), $\pi_{k, t}(m, n)$ is given as:

$\pi_{k, t}(m, 1)=\left(\begin{array}{c}m \\ k\end{array}\right) \cdot e^{-(k \sigma+1) t} \cdot\left(1-e^{-\sigma t}\right)^{m-k}$

Where $\mathrm{t}>0$ and $\mathrm{k}=0,1,2, \cdots, \mathrm{m}$;

For a particular case when $\sigma=1$, the value of $\pi_{k, t}(m, n)$ is given as:

$$
\pi_{k, t}(m, 1)=\left(\begin{array}{c}
m \\
k
\end{array}\right) \cdot e^{-(k+1) t} \cdot\left(1-e^{-t}\right)^{m-k}
$$

Where $\mathrm{t}>0$ and $\mathrm{k}=0,1,2, \cdots, \mathrm{m}$;

\section{Probability of Ultimate Number of Survivors}

Let $\pi_{k}(m, n)$ be the probability that k out of $\mathrm{m}$ susceptibles ultimately survive by the end of an epidemic initiated by $\mathrm{n}$ carriers. It can be obtained as the marginal distribution of $K$ as follows.

$\pi_{k}(m, n)=\int_{0}^{\infty}\left(\begin{array}{l}m \\ k\end{array}\right)\left(e^{-\sigma t}\right)^{k}\left(1-e^{-\sigma \mathrm{t}}\right)^{m-k} \frac{1}{\Gamma(n)} t^{n-1} e^{-t} d t$

Where $\mathrm{k}=0,1,2, \cdots, \mathrm{m}$;

Note: The above result obtained through our probabilistic approach is the same as that derived by Weiss ${ }^{27}$ from probability generating functions using a stochastic process approach.

Substituting $\mathrm{n}=1$ in equation (7), the expression for $\pi_{k}(m, 1)$ comes out to be:

$\pi_{k}(m, 1)=\int_{0}^{\infty}\left(\begin{array}{c}m \\ k\end{array}\right)\left(e^{-\sigma t}\right)^{k}\left(1-e^{-\sigma t}\right)^{m-k} e^{-t} d t=\frac{1}{\sigma} \cdot \frac{m !}{k !} \cdot \frac{\left(k+\frac{1}{\sigma}-1\right) !}{\left(m+\frac{1}{\sigma}-1\right) !}$

As a particular case, when the infection rate of susceptibles and the rate of removal of carriers are equal, i.e., the relative infection rate $\sigma=1$, we get.

$\pi_{k}(m, 1)=\frac{1}{m+1}$

Further, substituting $n=2$ in equation (7), the expression for $\pi_{k}(m, 2)$ comes out as:

$$
\begin{aligned}
& \pi_{k}(m, 2)=\int_{0}^{\infty}\left(\begin{array}{c}
m \\
k
\end{array}\right)\left(e^{-\sigma \mathrm{t}}\right)^{k}\left(1-e^{-\sigma \mathrm{t}}\right)^{m-k} t e^{-t} d t \\
& =\frac{1}{\sigma} \cdot \pi_{k}(m, 1) \cdot\left\{\frac{1}{k+\frac{1}{\sigma}}+\frac{1}{k+\frac{1}{\sigma}+1}+\cdots \cdot+\frac{1}{m+\frac{1}{\sigma}}\right\}
\end{aligned}
$$

Expressions of $\pi_{k}(m, n)$ for $n>2$ can also be derived from equation (7), or can be written in terms of polygamma functions tabulated by Davis. ${ }^{26}$ As a demonstration, values of $\pi_{k, t}(5,1)$ and $\pi_{k, t}(5,2)$ at different values of $k$ and $t$, and of $\pi_{k}(5,1), \pi_{k}(5,2), \pi_{k}(10,1)$ and $\pi_{k}(10,2)$, at different values of $k$, are computed and provided in Tables 3, 4, $5,6,7$ and 8 respectively for $\sigma=0.2$.

Further, graphs are plotted for these tabulated values to highlight the additional information provided by the plots of time-dependent survival probabilities as compared to the plots of only ultimate survival probabilities (Figures 1-6).

\section{Expectation and Variance of $\mathbf{K}$}

$$
\begin{aligned}
& \mathrm{E}(\mathrm{K})=\mathrm{E}[\mathrm{E}(\mathrm{K} \mid \mathrm{T}=\mathrm{t})]=\mathrm{E}\left[m \cdot e^{-\sigma \mathrm{t}}\right]=m \cdot E\left(e^{-\sigma \mathrm{t}}\right)=\frac{m}{(1+\sigma)^{n}} \\
& \mathrm{E}\left(K^{2}\right)=\mathrm{E}\left[\mathrm{E}\left(K^{2} \mid \mathrm{T}=\mathrm{t}\right)\right]=\mathrm{E}\left[m(m-1) \cdot e^{-2 \sigma \mathrm{t}}+m \cdot e^{-\sigma \mathrm{t}}\right] \\
& =m(m-1) \cdot E\left(e^{-2 \sigma \mathrm{t}}\right)+m \cdot E\left(e^{-\sigma \mathrm{t}}\right)=\frac{m(m-1)}{(1+2 \sigma)^{n}}+\frac{m}{(1+\sigma)^{n}} \\
& \operatorname{Var}(\mathrm{K})=\mathrm{E}\left(K^{2}\right)-(E[K])^{2}=\frac{m(m-1)}{(1+2 \sigma)^{n}}+\frac{m}{(1+\sigma)^{n}}-\left(\frac{m}{(1+\sigma)^{n}}\right)^{2}
\end{aligned}
$$


It can be observed that expectation and variance of $\mathrm{K}$ could be easily obtained by using our proposed method as compared to the method suggested by Wiess.

\section{Estimation of $\sigma$ (Relative Infection Rate)}

\section{Moment Estimator of $\sigma$}

Let $\mathrm{K}_{1} ; \mathrm{K}_{2} ; \ldots ; \mathrm{K}_{\mathrm{N}}$ be the random samples of susceptibles for various time periods from the distribution $\pi_{k}(m, n)$. By using the expression for the expectation of $K$ given in equation (11) and equating it to the sample mean $\mathrm{K}$ we have:

$\overline{\mathrm{K}}=\frac{m}{(1+\sigma)^{\mathrm{n}}}$; which gives $\hat{\sigma}_{m m e}=\left(\frac{m}{\mathrm{R}}\right)^{\frac{1}{n}}-1$

\section{Maximum Likelihood Estimator of $\sigma$}

Using the conditional distribution of $\mathrm{K}$ for given $\mathrm{T}=\mathrm{t}$, i.e., $\pi_{k \mid t}(m, n)$, the m.l.e. of $\sigma$ is obtained as:

$\widehat{e}^{-\sigma t}$ mle $(\mathrm{t})=\left(\frac{\overline{\mathrm{K}}}{m}\right)$ which implies $\hat{\sigma}_{m l e}(\mathrm{t})=\frac{1}{t} \cdot \ln \left(\frac{m}{\mathrm{~K}}\right)$; where $\mathrm{t}>0$ (15

If we replace $t$ by its expected value $n$ (i.e. $E(T)=n$, in the presence of $n$ carriers) then we get a specific m.l.e. of $\sigma$ as:

$$
\hat{\sigma}{ }^{*}{ }_{m l e}=\frac{1}{n} \cdot \ln \left(\frac{m}{\overline{\mathrm{K}}}\right)
$$

$\hat{\sigma} *_{m l e}$ would be an unbiased estimator of $\sigma$ (it is not possible to prove theoretically but we can prove numerically) and the variance of $\hat{\sigma} *{ }_{m l e}$ is:

$$
\operatorname{Var}\left(\widehat{\sigma}{ }_{m l e}\right)=\left[N \cdot m \cdot n^{2}\left\{1-\frac{1}{(1+\sigma)^{n}}\right\}\left(\frac{e^{-n \sigma}}{1+e^{-n \sigma}}\right)^{2}\right]^{-1}
$$

Where $\mathrm{N}$ is the number of sample observations on susceptibles from various time periods.

\section{Data Analysis}

\section{Check for efficiency of $\widehat{\boldsymbol{\sigma}} * \boldsymbol{m l}$}

We have applied our result on the data of typhoid epidemic in Zermatt, in which out of 1500 susceptibles approximately 100 cases of typhoid were reported. ${ }^{6,25}$

Our estimate $\hat{\sigma}{ }^{*}$ mle, the estimate $\hat{\sigma}^{\prime}$ mle of Roy Sunders and Richard J Kryscio ${ }^{25}$ and the estimate $\hat{\sigma}_{m m e}$ of Weiss ${ }^{6}$ for the data of typhoid epidemic in Zermatt are given below in Table 1.

Table I.Estimate of Relative Infection Rate ( $\sigma$ )

\begin{tabular}{|c|c|c|c|}
\hline $\mathbf{n}$ & $\mathbf{1}$ & $\mathbf{2}$ & $\mathbf{3}$ \\
\hline$\hat{\sigma}_{\text {mme }}$ & 0.071 & 0.035 & 0.023 \\
\hline$\hat{\sigma}^{*}{ }_{\text {mle }}$ & 0.070 & 0.034 & 0.023 \\
\hline$\hat{\sigma}^{\prime}{ }_{\text {mle }}$ & 0.067 & 0.033 & 0.022 \\
\hline
\end{tabular}

From Table 1 above, we observed that the numerical value of $\hat{\sigma}^{*}$ mle is very close to $\hat{\sigma}_{m m e}$ as compared to $\hat{\sigma}_{m l e}^{\prime}$.
For the same data, we now check the efficiency of our estimate $\hat{\sigma}{ }^{*}$ mle with $\hat{\sigma}^{\prime}$ mle of Roy Sunders and Richard J Kryscio, ${ }^{25}$ as shown below in Table 2.

\section{Table 2.Standard Error of Relative Infection Rate ( $\sigma$ )}

\begin{tabular}{|c|c|c|c|}
\hline $\mathbf{n}$ & $\mathbf{1}$ & $\mathbf{2}$ & $\mathbf{3}$ \\
\hline s.e. $\left(\hat{\sigma}^{*}{ }^{m l e}\right)$ & 0.037 & 0.019 & 0.012 \\
\hline s.e. $\left(\hat{\sigma}^{\prime}{ }^{m l e}\right)$ & 0.067 & 0.023 & 0.013 \\
\hline
\end{tabular}

Hence, from Table 2 it can be observed that $\hat{\sigma} *_{m l e}$ is more efficient as compared to $\hat{\sigma}_{m l e}^{\prime}$.

Finding Probability of $k$ Survivors with the help of $\pi_{k, t}(m, n)$ and $\pi_{k}(m, n)$

In Tables 3, 4, 5, 6, 7 and 8, the values of $\pi_{k, t}(5,1)$, $\pi_{k, t}(5,2), \pi_{k}(5,1), \pi_{k}(5,2), \pi_{k}(10,1)$ and $\pi_{k}(10,2)$, are presented for $\sigma=0.2$.

Table 3.Probability of $k$ Survivors for Weekly Time Periods when $\mathbf{n}=\mathbf{I}, \mathrm{m}$ $=5$ and $\sigma=0.2$

\begin{tabular}{|c|c|c|c|c|c|}
\hline$\pi_{k, t}(5,1)$ & $\mathbf{0 - 1}$ & $\mathbf{1 - 2}$ & $\mathbf{2}-\mathbf{3}$ & $\mathbf{3 - 4}$ & $\mathbf{4 - 5}$ \\
\hline $\mathbf{k}=0$ & 0.0000047 & 0.00026 & 0.00077 & 0.00098 & 0.00082 \\
\hline $\mathbf{k}=1$ & 0.00023 & 0.00373 & 0.00597 & 0.00815 & 0.00280 \\
\hline $\mathbf{k}=2$ & 0.000428 & 0.02132 & 0.01840 & 0.00950 & 0.00384 \\
\hline $\mathbf{k}=3$ & 0.04069 & 0.06094 & 0.02836 & 0.00937 & 0.00263 \\
\hline $\mathbf{k}=4$ & 0.19345 & 0.08709 & 0.02186 & 0.00462 & 0.00090 \\
\hline $\mathbf{k}=5$ & 0.36788 & 0.04979 & 0.00673 & 0.00091 & 0.00012 \\
\hline
\end{tabular}

Table 4.Probability of $k$ Survivors for Weekly Time Periods when $\mathrm{n}=\mathbf{2}, \mathrm{m}=5$ and $\sigma=0.2$

\begin{tabular}{|c|c|c|c|c|c|}
\hline$\pi_{k . t}(5,2)$ & $\mathbf{0 - 1}$ & $\mathbf{1 - 2}$ & $\mathbf{2 - 3}$ & $\mathbf{3 - 4}$ & $\mathbf{4 - 5}$ \\
\hline $\mathrm{k}=0$ & 0.0000023 & 0.00039 & 0.00194 & 0.00342 & 0.00368 \\
\hline $\mathrm{k}=1$ & 0.000123 & 0.00559 & 0.01492 & 0.01685 & 0.01261 \\
\hline $\mathrm{k}=2$ & 0.00214 & 0.03198 & 0.04599 & 0.03325 & 0.01727 \\
\hline $\mathrm{k}=3$ & 0.02035 & 0.09141 & 0.07089 & 0.03279 & 0.01183 \\
\hline $\mathrm{k}=4$ & 0.09673 & 0.13064 & 0.05464 & 0.01618 & 0.00405 \\
\hline $\mathrm{k}=5$ & 0.18394 & 0.07468 & 0.01684 & 0.00319 & 0.00056 \\
\hline
\end{tabular}

Table 5.Probability of $\mathbf{k}$ Survivors when $\mathbf{n}=\mathbf{I}, \mathbf{m}$ $=5$ and $\sigma=0.2$

\begin{tabular}{|c|c|c|c|c|c|c|}
\hline $\mathbf{k}$ & $\mathbf{0}$ & $\mathbf{1}$ & $\mathbf{2}$ & $\mathbf{3}$ & $\mathbf{4}$ & $\mathbf{5}$ \\
\hline$\pi_{k}(5,1)$ & 0.004 & 0.020 & 0.059 & 0.077 & 0.278 & 0.500 \\
\hline
\end{tabular}

Table 6.Probability of $k$ Survivors when $\mathbf{n}=2, \mathrm{~m}$ $=5$ and $\sigma=0.2$

\begin{tabular}{|c|c|c|c|c|c|c|}
\hline $\mathbf{k}$ & $\mathbf{0}$ & $\mathbf{1}$ & $\mathbf{2}$ & $\mathbf{3}$ & $\mathbf{4}$ & $\mathbf{5}$ \\
\hline$\pi_{k}(5,2)$ & 0.017 & 0.064 & 0.143 & 0.129 & 0.293 & 0.250 \\
\hline
\end{tabular}

Table 7.Probability of $\mathrm{k}$ Survivors when $\mathrm{n}=\mathrm{I}, \mathrm{m}=10$ and $\sigma=0.2$

\begin{tabular}{|c|c|c|c|c|c|c|c|c|c|c|c|}
\hline $\mathbf{k}$ & $\mathbf{0}$ & $\mathbf{1}$ & $\mathbf{2}$ & $\mathbf{3}$ & $\mathbf{4}$ & $\mathbf{5}$ & $\mathbf{6}$ & $\mathbf{7}$ & $\mathbf{8}$ & $\mathbf{9}$ & $\mathbf{1 0}$ \\
\hline$\pi_{k}(10,1)$ & 0.00033 & 0.002 & 0.005 & 0.012 & 0.023 & 0.042 & 0.077 & 0.110 & 0.165 & 0.238 & 0.333 \\
\hline
\end{tabular}


Table 8.Probability of $k$ Survivors when $n=2, m=10$ and $\sigma=0.2$

\begin{tabular}{|c|c|c|c|c|c|c|c|c|c|c|c|}
\hline $\mathrm{k}$ & 0 & 1 & 2 & 3 & 4 & 5 & 6 & 7 & 8 & 9 & 10 \\
\hline$\pi_{k}(10,2)$ & 0.002 & 0.011 & 0.022 & 0.043 & 0.069 & 0.103 & 0.150 & 0.164 & 0.177 & 0.164 & 0.111 \\
\hline
\end{tabular}

From the above tables, it is clear that as the time period and number of carriers increase, the chance of survival of susceptibles decreases. The graphical representation of $\pi_{k, t}(m, n)$ and $\pi_{k}(m, n)$ below also supports our results.

Graphical Representation of $\pi_{k, t}(m, n)$ and $\pi_{k}(m, n)$ for Table I to Table 8

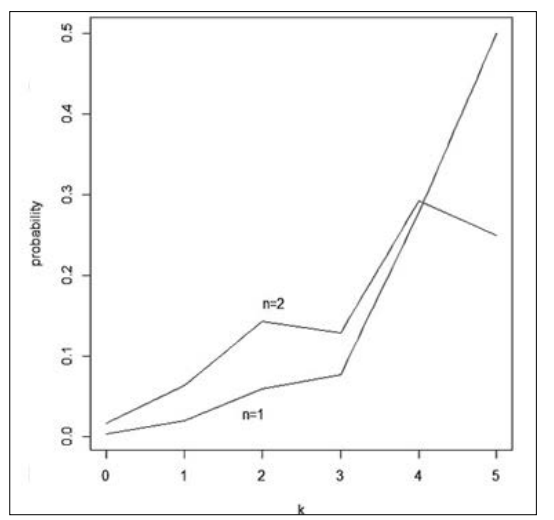

Figure I.Graphical Representation of $\pi_{k}(5, n)$ for $\sigma=0.2$

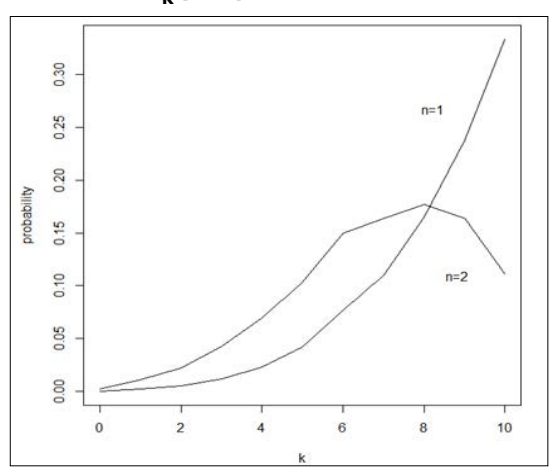

Figure 2.Graphical Representation of $\pi_{k}(10, n)$ for $\sigma=0.2$

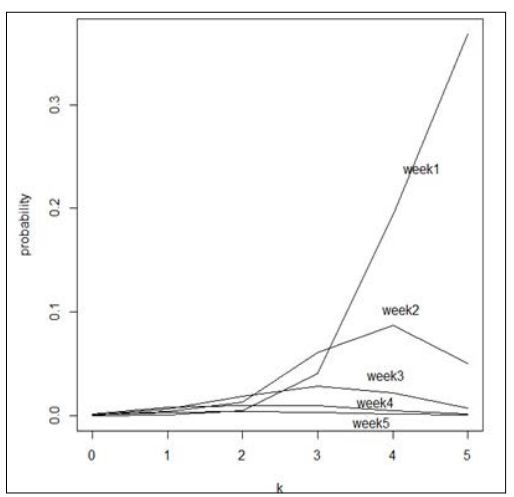

Figure 3.Graphical Representation of $\pi_{k, t}(5, I)$ for $\sigma=0.2$

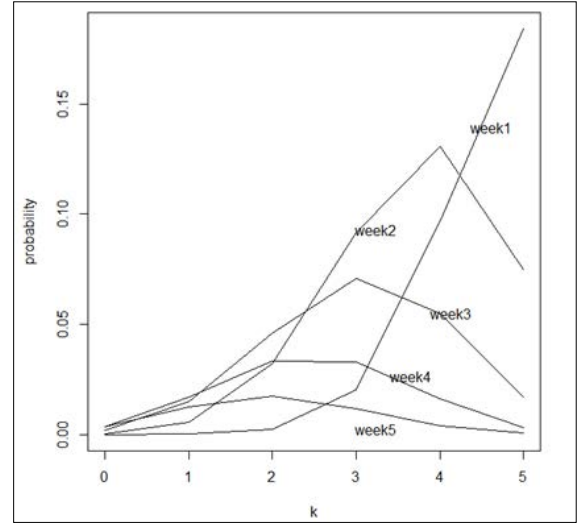

Figure 4.Graphical Representation of $\pi_{k, t}(5,2)$ for $\sigma=0.2$

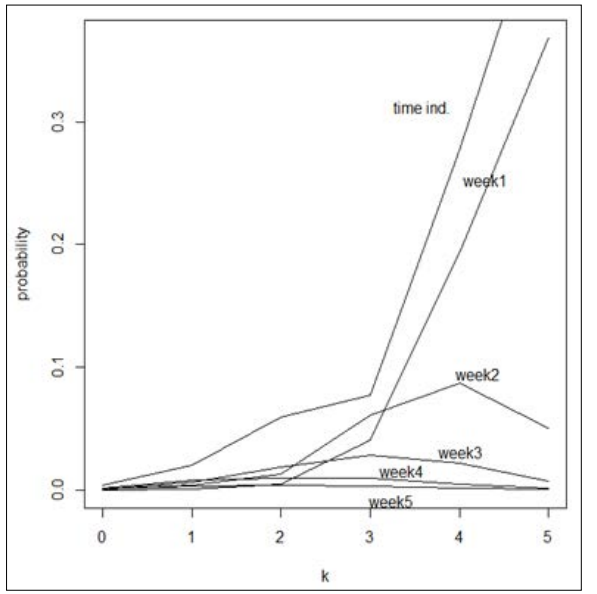

Figure 5.Graphical Representation of $\pi_{k, t}(5, I)$ vs $\pi_{k}(5, I)$ for $\sigma=0.2$

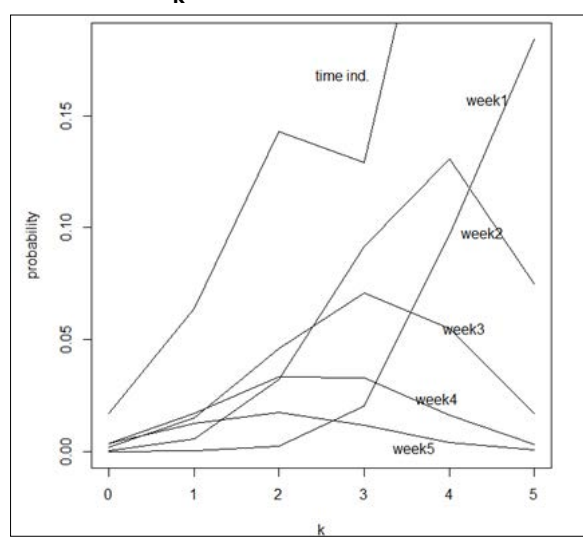

Figure 6. Graphical Representation of $\pi_{k, t}(5,2)$ vs $\pi_{\mathrm{k}}(5,2)$ for $\sigma=0.2$

\section{Discussion}

We have seen that the complete time-dependent theory gives a better explanation of the carrier-borne 
epidemic model than the stochastic theory. Also, it is observed that the infectious time period and the number of carriers play a significant role in the survival of susceptibles. From Figures 3 and 4, it is clear that the probability of survival of susceptibles decreases as the number of infectious time period increases. The probability to survive an infectious time period is smaller than that of the previous infectious time period. We can conclude from Figures 5 and 6 that the probability of survival of susceptibles based on stochastic theory is similar in nature to the probability survival of susceptibles at the first infectious time period (i.e., week 1) of time-dependent theory. The maximum likelihood estimate of $\sigma$ for time-dependent theory is easy to define but for stochastic theory, it is rather difficult. The MLE of $\sigma$ based on time-dependent theory is unbiased and more efficient than MLE of $\sigma$ based on stochastic theory suggested by Roy Sunders and Richard J Kryscio. ${ }^{25}$

\section{Conclusion}

We can conclude that the complete time-dependent theory explains the actual scenario of the carrierborne epidemic better than the stochastic theory. Also, it is observed that an increase in infectious time period will increase the number of carriers which plays a significant role in spreading infection among the susceptibles without showing the symptoms of the disease.

\section{Disclosure}

The authors have no conflict of interest to report.

\section{Funding}

The authors are grateful to the University Grants Commission of India for their financial support (JRF fellowship, UGC-Ref. No.:1082/(CSIR-UGC NET DEC.2018) in pursuing the research.

\section{Conflict of Interest: None}

\section{References}

1. Lotka AJ. Martini's equations for the epidemiology of immunizing diseases. Nature. 1923;111:633-4. [Google Scholar]

2. Ross R. The prevention of malaria. 2nd ed. London: John Murray; 1911.

3. Wilson GS. Measles as a universal disease. Am J Dis Child. 1962 Mar;103:219-23. [PubMed] [Google Scholar]

4. Dietz K. Introduction to Mckendrick (1926) Applications of mathematics to medical problems. In: Kotz S, Johnson NL, editors. Breakthroughs in Statistics. New York: Springer; 1926. [Google Scholar]

5. Bartlett MS. Some evolutionary stochastic processes. J Royal Stat Soci. 1949;11(2):211-29. [Google Scholar]

6. Weiss $\mathrm{GH}$. On the spread of epidemics by carriers. Biometrics. 1965 Jun;21:481-90. [PubMed] [Google Scholar]

7. Downton F. A note on the ultimate size of a general stochastic epidemic. Biometrica. 1967 Jun;54:3146. [PubMed] [Google Scholar]

8. Downton F. Epidemics with carriers - a note on a paper by Dietz. J Appl Prob. 1967;4:264-70. [Google Scholar]

9. Severo NC. The probabilities of some epidemic models. Biometrica. 1969;56:197-201. [Google Scholar]

10. Daniels HE. An exact relation in the theory of carrier-borne epidemics. Biometrica. 1972;59:2113. [Google Scholar]

11. Becker N. Carrier-borne epidemics in a community consisting of different groups. J Appl Prob. 1973;10:491-501. [Google Scholar]

12. Jerwood D. The cost of a carrier-borne epidemic. J Appl Prob. 1974;11:642-51. [Google Scholar]

13. Routleff C. A variation of Weiss's carrier-borne epidemic model. J Appl Prob. 1982;19(02):403-7. [Google Scholar]

14. Lefevre C, Malice MP. Comparisons for carrier borne epidemics in heterogenous and homogenous population. J Appl Prob. 1988;25(4). [Google Scholar]

15. Clancy D. Carrier-borne epidemic models incorporating population mobility. Math Biosci. 1996 Mar;132(2):185-204. [PubMed] [Google Scholar]

16. Dietz K. On the model of Weiss for the spread of epidemics by carriers. J Appl Prob. 1966;3:375-82. [Google Scholar]

17. Ball F. Deterministic and stochastic epidemics with several kinds of susceptibles. J Appl Prob. 1985;17:1-22. [Google Scholar]

18. Ball F, Clancy D. The final outcome and temporal solution of a carrier-borne epidemic model. J Appl Prob. 1985;32:304-15. [Google Scholar]

19. Gani J. On the general stochastic epidemic. Proc. 5th Berkeley Symp Math Statist Prob. 1967b;4:2719. [Google Scholar]

20. Gani J. A carrier-borne epidemic with two stages of infection. Comm Stat Stochs Model. 1991;7(1). [Google Scholar]

21. Picard P, Lefevre C. Another look at Gani's carrier- 
borne epidemic model with two stages of infection. Commun Stat. 1980;7:107-23. [Google Scholar]

22. Henderson W. A solution of the carrier-borne epidemic. J App Probability. 1979;16:641-5. [Google Scholar]

23. Watson R. On the size distribution for some epidemic models. J Appl Prob. 1980;17:912-21. [Google Scholar]

24. Bharucha-Reid AT. Elements of the theory of Markov process and their application. New York: McGraw-Hill; 1960. [Google Scholar]

25. Saunders R, Kryscio RJ. Parameter estimation for the carrier-borne epidemic model. J Royal Stat Soc. 1976;38(3):265-9. [Google Scholar]

26. Davis HT. Tables of the higher mathematical functions. Bloomington, IN: Principia Press; 1933. 